\title{
Reference Material (RM) 8820: A New Scanning Electron Microscope Scale Calibration Artifact
}

\author{
A. E. Vladár and M. T. Postek* \\ * National Institute of Standards and Technology, Gaithersburg, MD
}

NIST is introducing a new scanning electron microscope (SEM) scale calibration artifact called Reference Material (RM) 8820. RM 8820 is primarily intended to be used for calibrating the $\mathrm{X}$ and Y scale (or magnification) in SEMs from less than 10 times magnifications to more than 100000 times magnifications. Most SEMs require a set of calibration structures to cover the full range of possible magnifications and this standard is designed to meet that need. Like its predecessor RM 8090 , the new standard RM 8820 was designed to provide good contrast at both low and high electron landing energies (accelerating voltages). RM 8820 can also be used for non-linearity measurements, especially at lower than 10000 times magnifications. For these purposes, RM 8820 can be used on/in any other type of microscope, such as optical and scanning probe microscopes.

RM 8820 has been lithographically fabricated on a $20 \mathrm{~mm}$ x $20 \mathrm{~mm}$ silicon chip in collaboration with SEMATECH. The NIST pattern is a part of a very large array of test structures that were designed for various dimensional metrology purposes useful to semiconductor production technologies (see Figure). All the test structures are included on the RM. The RM 8820 parts of the chip are marked with letters "NIST" and are readily visible with the naked eye as small bright squares within the large chip. The RM structures have pitches ranging from $200 \mathrm{~nm}$ to $1.5 \mathrm{~mm}$ in both X and Y directions. In the center of the RM 8090 pattern, there is a large expanse of structures for focusing, astigmatism correction and for scan linearity measurements. There are two sets of identical $\mathrm{X}$ and $\mathrm{Y}$ pitch calibration structures. These are marked with numbers 1 and 3 for the $\mathrm{X}$ direction and 2 and 4 for the $\mathrm{Y}$ direction.

The RM 8090A samples were fabricated on $200 \mathrm{~mm}$ Si wafers using $193 \mathrm{~nm}$ ultraviolet light lithography and a dry etch process that formed all the patterns from an amorphous Si layer deposited on the silicon substrate with a thin silicon oxide layer in between. This $2 \mathrm{~nm}$ thin $\mathrm{SiO}_{2}$ was used as an etch stop. The average thickness of the amorphous Si layer was found to be $97.3 \mathrm{~nm}$ (with a standard deviation (STD) of $1.6 \mathrm{~nm}$ ). All amorphous silicon patterns exhibit a natural edge unevenness or edge roughness. This is due to the lithography and etch processes used in the fabrication of RM 8820. This edge roughness however does not have a very large effect on the pitch determination if a large enough number of pitches are considered in each pitch measurement.

The chip is intended to be mounted by the user on the proper stub, wafer or sample holder suitable for their particular instrument. Utmost care should be taken in the handling and mounting of the sample. Spring-loaded fasteners or very small amount of carbon conductive paste applied at two corners of the chip have been found to work well. In use, electron beam induced contamination will be deposited on the sample depending on the handling, instrument cleanliness, electron beam current and accelerating voltage used. Only the user, have control over these parameters, but it is possible to achieve cleanliness so that even after 10 minutes of continuous imaging there will be no visible change in the quality of the image and the measured value of the pitch. This may require the use of methods that are designed to clean the sample and the sample chamber and its vacuum system. 


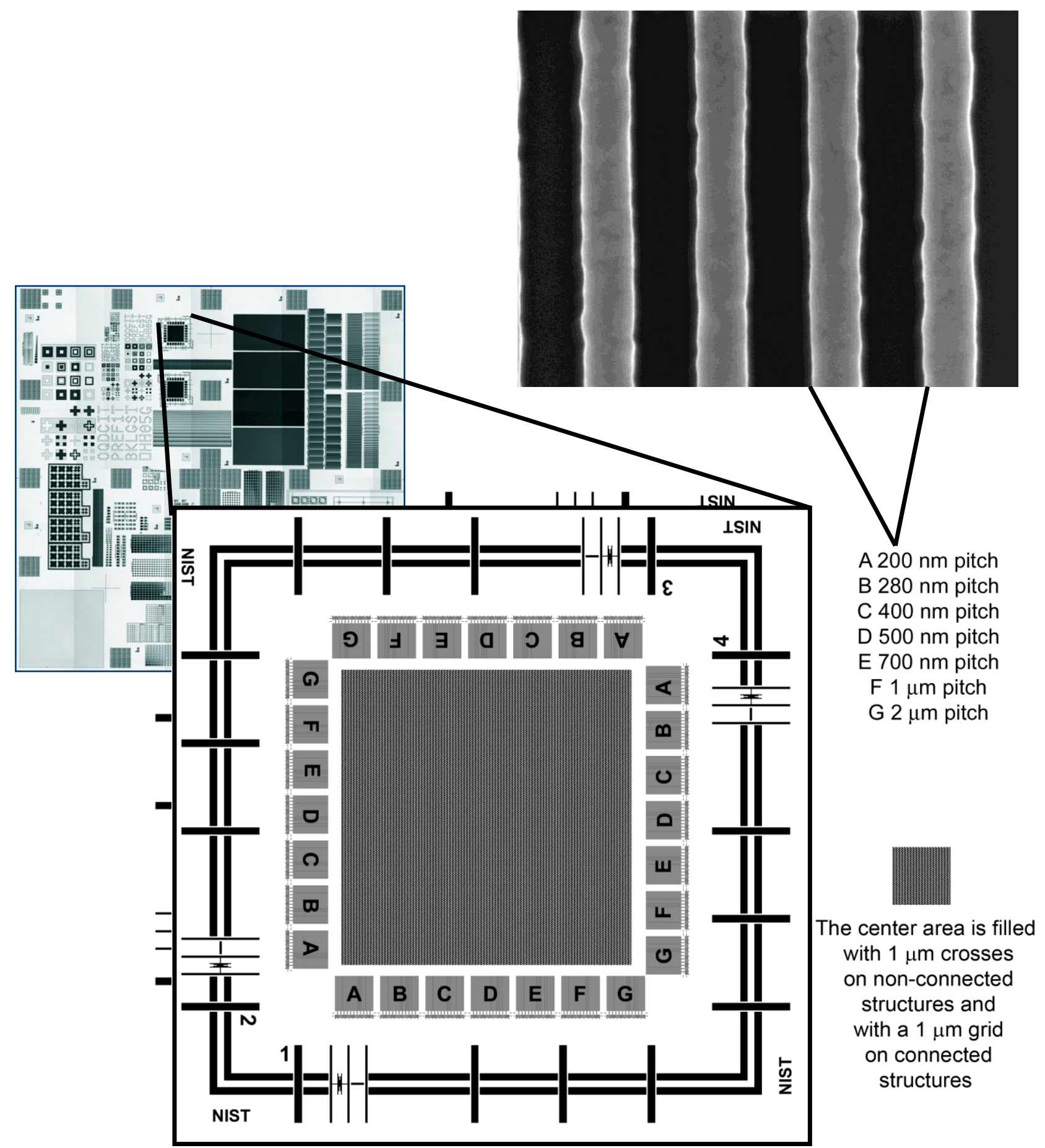

\section{References}

[1] Contribution of the National Institute of Standards and Technology; not subject to copyright.

[2] The authors would like to thank and acknowledge the collaboration of SEMATECH and the Advanced Metrology Advisory Group for assistance in the development of this standard and the NIST Office of Microelectronics Programs for partial funding of this work. 